\title{
A RELAXATION APPROXIMATION FOR DEGENERATE PARABOLIC EQUATIONS*
}

\author{
F. R. GUARGUAGLINI ${ }^{\dagger}$
}

\begin{abstract}
We present a semilinear relaxation approximation by hyperbolic-parabolic equations for quasilinear, possibly degenerate, parabolic problems, in several space dimensions. We prove the convergence of the approximated solutions to an entropy solution of the parabolic equation.
\end{abstract}

1. Introduction. We consider the Cauchy problem for a parabolic equation

$$
\left\{\begin{array}{l}
u_{t}+\sum_{j=1}^{d}\left(A_{j}(u)\right)_{x_{j}}-\Delta(B(u))=G(u) \\
u(x, 0)=u_{0}(x)
\end{array}\right.
$$

where $x=\left(x_{1}, \ldots, x_{d}\right) \in \mathbb{R}^{d}, u_{0} \in L^{1}\left(\mathbb{R}^{d}\right) \cap L^{\infty}\left(\mathbb{R}^{d}\right)$. We assume that $A_{j}, B$ and $G$ are smooth given functions and $B^{\prime}(u) \geq 0$.

In the purpose of approximate the solution $u$, we introduce the following semilinear system of equations

$$
\left\{\begin{array}{l}
\partial_{t} f_{i}^{\epsilon}+\sum_{j=1}^{d} \lambda_{j}^{i} \partial_{x_{j}} f_{i}^{\epsilon}-\mu^{i} \Delta f_{i}^{\epsilon}=\frac{1}{\epsilon}\left(M_{i}\left(u^{\epsilon}\right)-f_{i}^{\epsilon}\right)+\beta_{i} G\left(u^{\epsilon}\right), \quad i=1, \ldots, N \\
f_{i}^{\epsilon}(x, 0)=f_{0 i}^{\epsilon}(x), \quad i=1, \ldots, N
\end{array}\right.
$$

where

$$
u^{\epsilon}(x, t)=\sum_{i=1}^{N} f_{i}^{\epsilon}\left(x^{i}\right)
$$

and $\epsilon \in \mathbb{R}, \epsilon>0, \lambda^{i}=\left(\lambda_{1}^{i}, \ldots, \lambda_{d}^{i}\right) \in \mathbb{R}^{d}$ for $i=1, \ldots, N, \mu^{i} \in \mathbb{R}$ for $i=1, \ldots, N$, $\beta_{i} \in \mathbb{R}, \beta_{i} \geq 0$ for $i=1, \ldots, N, \sum_{i=1}^{N} \beta_{i}=1$ and $M=\left(M_{1}, \ldots, M_{N}\right) \in \operatorname{Lip}_{\text {loc }}\left(\mathbb{R} ; \mathbb{R}^{N}\right)$ with $M(0)=0$.

In this paper we want to show that, under the hypotesis that $f_{0 i}^{\epsilon}(x)=M_{i}\left(u_{0}\right)$, a subsequence of $\left\{u^{\epsilon}\right\}$ converges to a solution of problem (1.1) when $\epsilon \rightarrow 0$.

To this aim, we assume that $M$ is a Maxwellian Monotone Function (MMF) for system (1.2) in some interval $I \in \mathbb{R}$, i.e. $M$ satisfies the following conditions:

$$
\sum_{i=1}^{N} M_{i}(v)=v \quad \text { for every } v \in I
$$

$$
\sum_{i=1}^{N} \lambda_{j}^{i} M_{i}(v)=A_{j}(v) \quad \text { for every } v \in I, \quad j=1, \ldots, d
$$

$$
\sum_{i=1}^{N} \mu^{i} M_{i}(v)=B(v) \quad \text { for every } v \in I
$$

* Received April 14, 1998; revised Feb. 24, 1999.

† Dipartimento di Matematica Pura e Applicata, Universita' degli Studi di L'Aquila, Via Vetoio, loc. Coppito - 67100 L'Aquila, Italy (guarguag@univaq.it). Work partially supported by Istituto per le Applicazioni del Calcolo "M. Picone" - CNR - Viale del Policlinico 137, 00161 Rome, Italy and partially supported by TMR project HCL \# ERBFMRXCT960033. 
$\left(M_{4}\right) \quad M_{i}^{\prime}(v) \geq \max \left\{0,-\beta_{i} \epsilon_{0} G^{\prime}(v)\right\} \quad$ for every $v \in I, \quad i=1, \ldots, N, \quad \epsilon_{0}>0$.

The condition $\left(M_{4}\right)$ implies the quasimonotonicity of the right hand side of the equations in (1.2), for $\epsilon \leq \epsilon_{0}$; then special comparison and stability properties for system (1.2) are available ([7],[16]; see also [9], [13] and [14] for semilinear hyperbolic systems).

A system of type (1.2), with $M$ satisfying $\left(M_{1}\right)-\left(M_{4}\right)$, is similar to the BGK approximation for Euler equation (see [4]). We recall that the kinetic approximation of fluid dynamic equations is a classical problem in mathematical physics: the Euler equations can be formally obtained as the fluid dynamical limit of Boltzmann equations (see [4], [5] and references therein), but this limit has been rigorously established only as long as the limit solutions are regular. In the last years the rigorous theory of kinetic approximations for solutions with shocks, when the limit equation is scalar, has been developed. In [3] a convergence result of a fractional step continuous Boltzmann approximation to a parabolic (possibly degenerate) equation is proved. In [15] a continuous velocities BGK model is proved to be well adapted to describe general multidimensional scalar conservation laws as its microscopic scale, $\epsilon>0$, tends to zero. A related kinetic formulation of conservation laws can be found in [11]; moreover they suggest an extension of such formulation to parabolic (possibly degenerate) equations in conservation form.

We observe that the presence of the regions $\left\{x \in \mathbb{R}^{d}, t>0: B^{\prime}(u(x, t))=0\right\}$ makes the problem (1.1) of mixed type, in particular of hyperbolic-parabolic mixed type.

The porous media equation is an example of parabolic equation which degenerates in one point. Another kind of degeneracy has been investigated in the framework of "mushy" regions by [1].

The main features of our degenerate parabolic problem are the nonexistence of globally defined smooth solutions and the nonuniqueness of weak solutions, as in the pure quasilinear hyperbolic case.

It is well known that, for the hyperbolic case, uniqueness of solutions holds only in the class of functions verifiying special admissibility conditions called entropy inequalities [10].

In the same way, as a consequence of the possible degeneration to a hyperbolic problem, it is necessary to introduce the entropy inequalities for the equation in (1.1).

Definition 1.1. A function $u: \mathbb{R}^{d} \times[0, T] \rightarrow \mathbb{R}$ is an entropy solution of (1.1) if $u$ is a weak solution, $u$ verifies the inequalities

$$
\int_{0}^{T} \int_{\mathbb{R}^{d}}\left\{|u-k| \varphi_{t}+\operatorname{sgn}(u-k) \sum_{j=1}^{d}\left[\left(A_{j}(u)-A_{j}(k)\right] \varphi_{x_{j}}\right.\right.
$$

$$
+\operatorname{sgn}(u-k)[B(u)-B(k)] \Delta \varphi-\operatorname{sgn}(u-k) G(u) \varphi\} d x d t \geq 0,
$$

for all $k \in \mathbb{R}$ and for all $\varphi \in C_{0}^{\infty}\left(\mathbb{R}^{d} \times(0, T)\right), \varphi \geq 0$ and

$$
\lim _{t \rightarrow 0} \int_{\mathbb{R}^{d}}\left|u(x, t)-u_{0}(x)\right| d x=0 .
$$

Existence and uniqueness results for solutions, for the general case of equation (1.1), under different hypothesis, can be found in many papers, see [6] and reference 
therein; in particular we recall [17], [12] and [2]. In these papers the existence of an entropy solution is proved; however, only in the pure hyperbolic case the result about uniqueness is completely satisfactory [10]. More precisely, in the general case it is possible to obtain uniqueness for solutions which are limit of the vanishing viscosity method, but it is still an open problem whether or not it holds for all weak solutions satisfying conditions $(\mathrm{E})$. $^{*}$

The plan of this paper is as follows: in Section 2 we prove existence, uniqueness and stability for solutions to system (1.2). In Section 3 we show that a subsequence of $\left\{u^{\epsilon}\right\}$ converges in $C\left([0, T] ; L_{l o c}^{1}\left([0, T] \times \mathbb{R}^{d}\right)^{N}\right)$ to a solution of problem (1.1). Finally in Section 4 we present some relaxation systems to approximate problems belonging to the class (1.1).

Finally, let us remark that we may consider a more general class of parabolic (possibly degenerate) problems

$$
\left\{\begin{array}{l}
u_{t}+\sum_{j=1}^{d}\left(A_{j}(u)\right)_{x_{j}}-\sum_{j, k=1}^{d}\left(B_{j k}(u)\right)_{x_{j} x_{k}}=G(u) \\
u(x, 0)=u_{0}(x)
\end{array}\right.
$$

It is possible to prove that this equation can be approximated by means of the following system of semilinear equations

$$
\left\{\begin{array}{r}
\partial_{t} f_{i}^{\epsilon}+\sum_{j=1}^{d} \lambda_{j}^{i} \partial_{x_{j}} f_{i}^{\epsilon}-\sum_{j, k=1}^{d} \mu_{j k}^{i} \partial_{x_{j} x_{k}} f_{i}^{\epsilon}=\frac{1}{\epsilon}\left[M_{i}\left(u^{\epsilon}\right)-f_{i}^{\epsilon}\right]+\beta_{i} G\left(u^{\epsilon}\right), \\
i=1, \ldots, N \\
f_{i}^{\epsilon}(x, 0)=f_{0 i}^{\epsilon}(x) i=1, \ldots, N,
\end{array}\right.
$$

where $u^{\epsilon}(x, t)=\sum_{i=1}^{N} f_{i}^{\epsilon}(x, t)$ and $\left(\mu^{i}\right)_{j k}$ are $d \times d$ real positive definite matrices. The techniques of the present paper can be easily extended to cover this case.

2. Existence, uniqueness, stability for the system. In this section we prove existence, uniqueness and $L^{1}$-stability for the solutions of system (1.2).

Through the whole paper we assume that the following conditions are satisfied:

i) $A_{j} \in \operatorname{Lip}_{\text {loc }}(\mathbb{R} ; \mathbb{R})$ for $j=1, \ldots, d$;

ii) $B \in \operatorname{Lip}_{\text {loc }}(\mathbb{R} ; \mathbb{R})$ and $B^{\prime}(u) \geq 0$;

iii) $G \in \operatorname{Lip}_{\text {loc }}(\mathbb{R} ; \mathbb{R})$ and, for initial data in some interval $J \subseteq \mathbb{R}$, the differential equation $\dot{w}=G(w)$ admits global solutions.

Moreover we assume that $\pm\left\|u_{0}\right\|_{L^{\infty}(\mathbb{R})} \in J$.

Set $\|f(\cdot, t)\|_{L^{\infty}\left(\mathbb{R}^{d}\right)^{N}}:=\sum_{i=1}^{N}\left\|f_{i}(\cdot, t)\right\|_{L^{\infty}\left(\mathbb{R}^{d}\right)}$.

Proposition 2.1 (Local EXistence And Uniqueness). Let $f_{0 i}^{\epsilon} \in L^{\infty}\left(\mathbb{R}^{d}\right) \cap$ $L^{1}\left(\mathbb{R}^{d}\right)$, for $i=1, \ldots, N$ and $\epsilon>0$ and fix $V \in \mathbb{R}$ such that $V>\left\|f_{0}^{\epsilon}\right\|_{L^{\infty}\left(\mathbb{R}^{d}\right)^{N}}$. Assume that $M$ is a $M M F$ on the interval $I:=\{u \in \mathbb{R}:|u| \leq V\}$. Then for every $\epsilon>0$ there exists a unique local weak solution $f^{\epsilon} \in C\left([0, \tau] ; L^{\infty}\left(\mathbb{R}^{d}\right)^{N}\right)$ to the system (1.2).

Proof. We can assume that $\mu^{i}>0$ for $i=1, \ldots, m$ and $\mu^{i}=0$ for $i=m+1, \ldots, N$. Let

$$
K_{i}(x, t):=\frac{1}{\sqrt{4 \pi \mu^{i} t}} \exp \left(-\frac{\|x\|_{\mathbb{R}^{N}}}{4 \mu^{i} t}\right) .
$$

\footnotetext{
* See however the recent results in [18].
} 
A function $f^{\epsilon}$ is a solution of the system (1.2) if and only if for $i=1, \ldots, m, f_{i}^{\epsilon}$ is given by the Green's formula

$$
\begin{gathered}
f_{i}^{\epsilon}(x, t)=e^{-\frac{t}{\epsilon}} \int_{\mathbb{R}^{d}} K_{i}\left(x-\lambda^{i} t-y, t\right) f_{0 i}^{\epsilon}(y) d y \\
+\frac{1}{\epsilon} \int_{0}^{t} e^{-\frac{t-s}{\epsilon}} \int_{\mathbb{R}^{d}} K_{i}\left(x-\lambda^{i} t-y, t-s\right) M_{i}\left(u^{\epsilon}\left(y+\lambda^{i} s, s\right)\right) d y d s \\
+\beta_{i} \int_{0}^{t} e^{-\frac{t-s}{\epsilon}} \int_{\mathbb{R}^{d}} K_{i}\left(x-\lambda^{i} t-y, t-s\right) G\left(u^{\epsilon}\left(y+\lambda^{i} s, s\right) d y d s\right.
\end{gathered}
$$

and for $i=m+1, \ldots, N, f_{i}^{\epsilon}$ is given by the Duhamel's formula

$$
\begin{aligned}
f_{i}^{\epsilon}(x, t) & =e^{-\frac{t}{\epsilon}} f_{0 i}^{\epsilon}\left(x-\lambda^{i} t\right) \\
+ & +\frac{1}{\epsilon} \int_{0}^{t} e^{-\frac{t-s}{\epsilon}} M_{i}\left(u^{\epsilon}\left(x-\lambda^{i}(t-s), s\right)\right) d s \\
& +\beta_{i} \int_{0}^{t} e^{-\frac{t-s}{\epsilon}} G\left(u^{\epsilon}\left(x-\lambda^{i}(t-s), s\right)\right) d s .
\end{aligned}
$$

Consider the Banach space

$$
S_{\tau}:=\left\{f \in C\left([0, \tau] ; L^{\infty}\left(\mathbb{R}^{d}\right)^{N}\right): \sup _{t \in[0, \tau]}\|f(\cdot, t)\|_{L^{\infty}\left(\mathbb{R}^{d}\right)^{N}} \leq V\right\}
$$

and the operator $\phi^{\epsilon}$ defined on $S_{\tau}$ as follows: for $i=1, \ldots, m$,

$$
\begin{aligned}
& \phi_{i}^{\epsilon}(f)(x, t)=e^{-\frac{t}{\epsilon}} \int_{\mathbb{R}^{d}} K_{i}\left(x-\lambda^{i} t-y, t\right) f_{0 i}(y) d y \\
& +\frac{1}{\epsilon} \int_{0}^{t} e^{-\frac{t-s}{\epsilon}} \int_{\mathbb{R}^{d}} K_{i}\left(x-\lambda^{i} t-y, t-s\right) M_{i}\left(w\left(y+\lambda^{i} s, s\right)\right) d y d s \\
& \quad+\beta_{i} \int_{0}^{t} e^{-\frac{t-s}{\epsilon}} \int_{\mathbb{R}^{d}} K_{i}\left(x-\lambda^{i} t-y, t-s\right) G\left(w\left(y+\lambda^{i} s, s\right) d y d s\right.
\end{aligned}
$$

for $i=m+1, \ldots, N$,

$$
\begin{aligned}
\phi_{i}^{\epsilon}(f)(x, t)= & e^{-\frac{t}{\epsilon}} f_{0 i}\left(x-\lambda^{i} t\right) \\
& +\frac{1}{\epsilon} \int_{0}^{t} e^{-\frac{t-s}{\epsilon}} M_{i}\left(w\left(x-\lambda^{i}(t-s), s\right)\right) d s \\
& \quad+\beta_{i} \int_{0}^{t} e^{-\frac{t-s}{\epsilon}} G\left(w\left(x-\lambda^{i}(t-s), s\right)\right) d s,
\end{aligned}
$$

where $w:=\sum_{i=1}^{N} f_{i}$.

We want to prove that for every $\epsilon>0$, there exists $\tau>0$ sufficiently small such that $\phi^{\epsilon}$ is a contraction from $S_{\tau}$ in $S_{\tau}$. Using the assumptions on the functions $M$ and $G$ we obtain

$$
\begin{gathered}
\sup _{t \in[0, \tau]}\left\|\phi^{\epsilon}(f)(\cdot, t)\right\|_{L^{\infty}\left(R^{d}\right)^{N}} \leq\left\|f_{0}^{\epsilon}\right\|_{L^{\infty}\left(R^{d}\right)^{N}} \\
+\left(1-e^{-\frac{\tau}{\epsilon}}\right)\left(N+\epsilon L_{V}\right) V
\end{gathered}
$$


where $L_{V}$ is the Lipschitz constant of $G$ in $[-V, V]$. Hence, for every $\epsilon>0$ there exists a value $\tau$ sufficiently small such that $\phi^{\epsilon}\left(S_{\tau}\right) \subseteq S_{\tau}$.

In a similar way we obtain that, if $f_{1}, f_{2} \in S_{\tau}$, then

$$
\begin{gathered}
\left\|\phi^{\epsilon}\left(f_{1}\right)(\cdot, t)-\phi^{\epsilon}\left(f_{2}\right)(\cdot, t)\right\|_{L^{\infty}\left(R^{d}\right)^{N}} \\
\leq\left(1-e^{-\frac{\tau}{\epsilon}}\right)\left(1+\epsilon L_{V}\right) \sup _{t \in[0, \tau]}\left\|f_{1}(\cdot, t)-f_{2}(\cdot, t)\right\| \|_{L^{\infty}\left(R^{d}\right)^{N}}
\end{gathered}
$$

therefore, for $\tau$ sufficiently small, $\phi^{\epsilon}$ is a contraction in $S_{\tau}$ and the conclusion follows.

Proposition 2.2 ( $L^{1}$ Stability). Let $\epsilon>0, f_{0 i}^{\epsilon}, \overline{f_{0 i}^{\epsilon}} \in L^{\infty}\left(\mathbb{R}^{d}\right) \cap L^{1}\left(\mathbb{R}^{d}\right) ;$ let $f^{\epsilon}, \overline{f^{\epsilon}} \in C\left([0, T] ; L^{\infty}\left(R^{d}\right)^{N}\right)$ be the corresponding solutions of the equation in (1.2), for some $T>0$, and

$$
V:=\max \left\{\sup _{t \in[0, T]}\left\|f^{\epsilon}(\cdot, t)\right\|_{L^{\infty}\left(\mathbb{R}^{d}\right)^{N}} \sup _{t \in[0, T]}\left\|\overline{f^{\epsilon}}(\cdot, t)\right\|_{L^{\infty}\left(\mathbb{R}^{d}\right)^{N}}\right\} .
$$

Assume that $M$ is a $M M F$ on the interval $I:=\{u \in \mathbb{R}:|u| \leq V\}$. Then for $0 \leq s \leq t \leq T$

$$
\sum_{i=1}^{N} \int_{\mathbb{R}^{d}}\left|f_{i}^{\epsilon}(x, t)-\overline{f_{i}^{\epsilon}}(x, t)\right| d x \leq e^{L_{V}(t-s)} \sum_{i=1}^{N} \int_{R^{d}}\left|f_{i}^{\epsilon}(x, s)-\overline{f_{i}^{\epsilon}}(x, s)\right| d x,
$$

where $L_{V}$ is the Lipschitz constant of $G$ in $[-V, V]$.

Proof. From (2.1) and (2.2), multiplying for the function $\operatorname{sgn}\left(f_{i}^{\epsilon}-\overline{f_{i}^{\epsilon}}\right)$, integrating on $\mathbb{R}^{d}$ and summing up for $i=1, \ldots, N$, we obtain

$$
\begin{aligned}
\sum_{i=1}^{N} \int_{\mathbb{R}^{d}}\left|f_{i}^{\epsilon}(x, t)-\overline{f_{i}^{\epsilon}}(x, t)\right| d x \leq e^{-\frac{t-s}{\epsilon}} \sum_{i=1}^{N} \int_{\mathbb{R}^{d}}\left|f_{i}^{\epsilon}(x, s)-\overline{f_{i}^{\epsilon}}(x, s)\right| d x \\
+\frac{1}{\epsilon} \int_{s}^{t} e^{-\frac{t-\tau}{\epsilon}} \int_{R^{d}} \mid M_{i}\left(u^{\epsilon}(x, \tau)\right)-M_{i}\left(\overline{u^{\epsilon}}(x, \tau) \mid d x d \tau\right. \\
+\beta_{i} \int_{s}^{t} e^{-\frac{t-\tau}{\epsilon}} \int_{R^{d}} \mid G\left(u^{\epsilon}(x, \tau)\right)-G\left(\overline{u^{\epsilon}}(x, \tau) \mid d x d \tau .\right.
\end{aligned}
$$

Then, setting for $0 \leq s \leq t$

$$
\rho(s):=\int_{R^{d}} \sum_{i=1}^{N}\left|f_{i}^{\epsilon}(x, s)-\overline{f_{i}^{\epsilon}}(x, s)\right| d x,
$$

we have

$$
\rho(t) \leq e^{-\frac{t-s}{\epsilon}} \rho(s)+\left(\frac{1}{\epsilon}+B\right) \int_{s}^{t} e^{-\frac{t-\tau}{\epsilon}} \rho(\tau) d \tau .
$$

From the above inequality we obtain

$$
\rho(t) \leq e^{L_{V}(t-s)} \rho(s) \quad \text { for } 0 \leq s \leq t
$$

which concludes the proof.

In order to obtain the existence and uniqueness of a solution $f \in$ $C\left([0, T] ; L^{\infty}\left(\mathbb{R}^{d}\right)^{N} \cap L^{1}\left(\mathbb{R}^{d}\right)^{N}\right)$ to problem $(1.2)$, for all $T>0$, we need to prove a 
comparison result. First we will prove comparison, existence and uniqueness results for smooth solutions, then we will extend to solutions in $C\left([0, T] ; L^{\infty}\left(\mathbb{R}^{d}\right)^{N} \cap L^{1}\left(\mathbb{R}^{d}\right)^{N}\right)$ by density arguments.

Proposition 2.3 (Comparison For ClASSICAL SOluTions). Let $\epsilon>0, T>0$ and $f_{0 i}^{\epsilon}, \overline{f_{0 i}^{\epsilon}} \in C_{0}^{\infty}\left(\mathbb{R}^{d}\right)$; let $f^{\epsilon}, \overline{f^{\epsilon}}$ be the corresponding solutions of the system in (1.2), in $\mathbb{R}^{d} \times[0, T]$ and

$$
V:=\max \left\{\sup _{t \in[0, T]}\left\|f^{\epsilon}(\cdot, t)\right\|_{L^{\infty}\left(\mathbb{R}^{d}\right)^{N}} \sup _{t \in[0, T]}\left\|\overline{f^{\epsilon}}(\cdot, t)\right\|_{L^{\infty}\left(\mathbb{R}^{d}\right)^{N}}\right\} .
$$

Assume that $M$ is a MMF on the interval $I:=\{u \in \mathbb{R}:|u| \leq V\}$. Then for $0 \leq t \leq T$ and for $i=1, \ldots, N$,

$$
\begin{aligned}
& \sum_{i=1}^{N} \int_{\mathbb{R}^{d}}\left[f_{i}^{\epsilon}(x, t)-\overline{f_{i}^{\epsilon}}(x, t)\right]_{+} d x \\
& \leq e^{\left[(N-1) \gamma^{+}-\gamma\right] t} \sum_{i=1}^{N} \int_{\mathbb{R}^{d}}\left[f_{0 i}^{\epsilon}(x)-\overline{f_{0 i}^{\epsilon}}(x)\right]_{+} d x
\end{aligned}
$$

where

$$
\gamma:=\max _{1 \leq i \leq N}\left\|\frac{\partial\left(\frac{1}{\epsilon}\left(M_{i}\left(u^{\epsilon}\right)-f_{i}^{\epsilon}\right)+\beta_{i} G\left(u^{\epsilon}\right)\right)}{\partial f_{i}^{\epsilon}}\right\|_{L^{\infty}([-V, V])}
$$

and

$$
\gamma^{+}:=\max _{1 \leq i, j \leq N, i \neq j}\left\|\frac{\partial\left(\frac{1}{\epsilon}\left(M_{i}\left(u^{\epsilon}\right)-f_{i}^{\epsilon}\right)+\beta_{i} G\left(u^{\epsilon}\right)\right)}{\partial f_{j}^{\epsilon}}\right\|_{L^{\infty}([-V, V])} .
$$

Proof. We set

$$
Q_{i}^{\epsilon}\left(u^{\epsilon}\right):=\frac{1}{\epsilon}\left(M_{i}\left(u^{\epsilon}\right)-f_{i}^{\epsilon}\right)+\beta_{i} G\left(u^{\epsilon}\right)
$$

and

$$
H^{+}(y)=\frac{1}{2}(\operatorname{sgn} y+1) .
$$

Multiplying for $H^{+}\left(f_{i}^{\epsilon}-\overline{f_{i}^{\epsilon}}\right)$ the equations for $f_{i}^{\epsilon}$ and $\overline{f_{i}^{\epsilon}}$, making the difference and integrating on $\mathbb{R}^{d}$ we obtain

$$
\begin{gathered}
\int_{\mathbb{R}^{d}}\left(f_{i}^{\epsilon}-\overline{f_{i}^{\epsilon}}\right)_{t} H^{+}\left(f_{i}^{\epsilon}-\overline{f_{i}^{\epsilon}}\right) d x+\sum_{j=1}^{d} \lambda_{j}^{i} \int_{\mathbb{R}^{d}}\left(f_{i}^{\epsilon}-\overline{f_{i}^{\epsilon}}\right)_{x_{j}} H^{+}\left(f_{i}^{\epsilon}-\overline{f_{i}^{\epsilon}}\right) d x \\
-\sum_{j=1}^{d} \mu^{i} \int_{\mathbb{R}^{d}}\left(f_{i}^{\epsilon}-\overline{f_{i}^{\epsilon}}\right)_{x_{j} x_{j}} H^{+}\left(f_{i}^{\epsilon}-\overline{f_{i}^{\epsilon}}\right) d x=\int_{\mathbb{R}^{d}} H^{+}\left(f_{i}^{\epsilon}-\overline{f_{i}^{\bar{\epsilon}}}\right)\left(Q_{i}^{\epsilon}\left(u^{\epsilon}\right)-Q_{i}^{\epsilon}\left(\overline{u^{\epsilon}}\right)\right) d x .
\end{gathered}
$$

Then we have

$$
\begin{aligned}
\int_{\mathbb{R}^{d}}\left(\left[f_{i}^{\epsilon}-\overline{f_{i}^{\epsilon}}\right]_{+}\right)_{t} d x & \leq \int_{\mathbb{R}^{d}}\left(\left[f_{0 i}^{\epsilon}-\overline{f_{0 i}^{\epsilon}}\right]_{+}\right)_{t} d x \\
& +\int_{0}^{t} \int_{\mathbb{R}^{d}} H^{+}\left(f_{i}^{\epsilon}-\overline{f_{i}^{\epsilon}}\right)\left(Q_{i}\left(u^{\epsilon}\right)-Q_{i}\left(\overline{u^{\epsilon}}\right)\right) d x d t .
\end{aligned}
$$


Now we go on following [9]. Using the quasimonotonicity of $Q^{\epsilon}$ we obtain

$$
\begin{aligned}
& \sum_{i=1}^{N} H^{+}\left(f_{i}^{\epsilon}-\overline{f_{i}^{\epsilon}}\right)\left(Q_{i}^{\epsilon}\left(u^{\epsilon}\right)-Q_{i}^{\epsilon}\left(\overline{u^{\epsilon}}\right)\right. \\
& \leq \sum_{i=1}^{N} \sum_{j=1 j \neq i}^{N} \gamma^{+}\left[f_{i}^{\epsilon}-\overline{f_{i}^{\epsilon}}\right]_{+}+\gamma \sum_{i=1}^{N}\left[f_{i}^{\epsilon}-\overline{f_{i}^{\epsilon}}\right]_{+} \\
& \quad=\left((N-1) \gamma^{+}+\gamma\right) \sum_{i=1}^{N}\left[f_{i}^{\epsilon}-\overline{f_{i}^{\epsilon}}\right]_{+} .
\end{aligned}
$$

If we set

$$
\rho(t):=\sum_{i=1}^{N} \int_{\mathbb{R}^{d}}\left[f_{i}^{\epsilon}-\overline{f_{i}^{\epsilon}}\right]_{+} d x
$$

from

$$
\rho(t) \leq \rho(0)+\left((N-1) \gamma^{+}-\gamma\right) \int_{0}^{t} \rho(s) d s
$$

we obtain

$$
\rho(t) \leq e^{\left[(N-1) \gamma^{+}-\gamma\right] t} \rho(0) .
$$

Now the conclusion follows.

From the above proposition we immediately obtain the following result.

Corollary 2.1. Under the hypothesys of Proposition 2.3, if

$$
f_{0 i}^{\epsilon}(x) \leq \overline{f_{0 i}^{\epsilon}}(x) \text { a.e. in } \mathbb{R}^{d},
$$

then

$$
f_{i}^{\epsilon}(x, t) \leq \overline{f_{i}^{\epsilon}}(x, t) \text { a.e. in } \mathbb{R}^{d} \times[0, T]
$$

Now we can prove an $L^{\infty}$ estimate, uniform in $\epsilon$, for classical solutions of (1.2). The proof is based on comparison with the solutions of the ordinary differential problems

$$
\left\{\begin{array}{l}
\dot{w}^{ \pm}=G(w), \\
w^{ \pm}(0)= \pm\left\|u_{0}\right\|_{L^{\infty}\left(R^{d}\right)} .
\end{array}\right.
$$

The assumptions made at the beginning of the section ensure that, for such initial data, the differential equation admits global solutions .

Let $T>0$ and

$$
V_{T}:=\max \left\{\max _{0 \leq t \leq T}\left|w^{+}\right|, \max _{0 \leq t \leq T}\left|w^{-}\right|\right\}
$$

Proposition 2.4 ( $L^{\infty}$ estimate for Classical solutions). Let $T>0$, let $V_{T}$ be the constant defined above and $M$ be a MMF in $\left[-V_{T}, V_{T}\right]$. Let $\epsilon>0$, $f_{0 i}^{\epsilon} \in C_{0}^{\infty}\left(\mathbb{R}^{d}\right)$ and $f_{i}^{\epsilon}$ be the corresponding solution of the problem (1.2). Then

$$
\begin{gathered}
\left\|u^{\epsilon}(\cdot, t)\right\|_{L^{\infty}\left(\mathbb{R}^{d}\right)} \leq V_{T}, \quad 0 \leq t \leq T \\
-C_{i T}+M_{i}\left(-\left\|u_{0}\right\|_{L^{\infty}\left(\mathbb{R}^{d}\right)}\right) \leq f_{i}^{\epsilon}(x, t) \leq C_{i T}+M_{i}\left(\left\|u_{0}\right\|_{L^{\infty}\left(\mathbb{R}^{d}\right)}\right), \quad 0 \leq t \leq T,
\end{gathered}
$$


for $i=1, \ldots, N$, where $C_{i T}:=V_{T}+\beta_{i} \max _{\left[-V_{T}, V_{T}\right]}|G|$.

Proof. Set

$$
M_{i}^{*}(u)=\left\{\begin{array}{lc}
M_{i}(-V) & u<-V, \\
M_{i}(u) & |u| \leq V, \\
M_{i}(V) & u>V,
\end{array}\right.
$$

for $i=1, \ldots, N$; let $f^{\epsilon *}$ be the solution of system (1.2) with $M$ replaced by $M^{*}$ and $u^{\epsilon *}:=\sum_{i=1}^{N} f_{i}^{\epsilon *}$.

We consider the ordinary differential problem

$$
\left\{\begin{array}{l}
\dot{p}_{\epsilon i}^{ \pm}(t)=\frac{1}{\epsilon}\left(M_{i}^{*}\left(v_{\epsilon}^{ \pm}\right)-p_{\epsilon i}^{ \pm}\right)+\beta_{i} G\left(v_{\epsilon}^{ \pm}\right), \quad 0 \leq t \leq T \\
p_{\epsilon i}^{ \pm}(0)=M_{i}\left( \pm\left\|u_{0}\right\|_{L^{\infty}\left(R^{d}\right)}\right) \\
v_{\epsilon}^{ \pm}:=\sum_{i=1}^{N} p_{\epsilon i}^{ \pm}
\end{array}\right.
$$

The assumptions on $M^{*}$ imply that

$$
\begin{array}{ll}
\dot{v}_{\epsilon}^{ \pm}=G\left(v_{\epsilon}^{ \pm}\right) & 0 \leq t \leq T, \\
\left|v_{\epsilon}^{ \pm}(t)\right| \leq V_{T} & 0 \leq t \leq T .
\end{array}
$$

Now, due to the quasimonotonicity of the system (1.2), we can use the comparison result in Corollary 2.1 to obtain

$$
\begin{array}{ll}
p_{\epsilon i}^{-} \leq f_{i}^{\epsilon^{*}} \leq p_{\epsilon i}^{+} & (x, t) \in \mathbb{R}^{d} \times[0, T], \\
v_{\epsilon}^{-} \leq u^{\epsilon^{*}} \leq v_{\epsilon}^{+} & (x, t) \in \mathbb{R}^{d} \times[0, T] .
\end{array}
$$

We immediately have that

$$
\left\|u^{\epsilon^{*}}(\cdot, t)\right\|_{L^{\infty}\left(R^{d}\right)} \leq V_{T} \quad 0 \leq t \leq T .
$$

Moreover, using again the assumptions on $M^{*}$, we have

$$
\begin{aligned}
\dot{p}_{\epsilon i}^{+}(t) \leq \frac{1}{\epsilon}\left(M_{i}^{*}\left(V_{T}\right)-p_{\epsilon i}^{+}\right)+\beta_{i} \max _{\left[-V_{T}, V_{T}\right]}|G| \\
\leq \frac{1}{\epsilon}\left(V_{T}-p_{\epsilon i}^{+}\right)+\beta_{i} \max _{\left[-V_{T}, V_{T}\right]}|G|
\end{aligned}
$$

which implies

$$
p_{\epsilon i}^{+}(t) \leq V_{T}+\beta_{i} \max _{\left[-V_{T}, V_{T}\right]}|G|+M_{i}\left(\left\|u_{0}\right\|_{L^{\infty}\left(R^{d}\right)}\right) .
$$

In a similar way we obtain

$$
p_{\epsilon i}^{-}(t) \geq-\left(V_{T}+\beta_{i} \max _{\left[-V_{T}, V_{T}\right]}|G|-M_{i}\left(-\left\|u_{0}\right\|_{L^{\infty}\left(R^{d}\right)}\right)\right) .
$$

Now, on the range of $u^{\epsilon^{*}}, M=M^{*}$ and the claim follows.

The above results imply that, for smooth initial data, for every $\epsilon>0$ and for all $T>0$ there exists a unique solution $f^{\epsilon} \in C\left([0, T] ; L^{\infty}\left(\mathbb{R}^{d}\right)^{N} \cap L^{1}\left(\mathbb{R}^{d}\right)^{N}\right)$ to problem (1.2).

By standard density argument we can extend the result to the case of initial data in $L^{\infty}\left(\mathbb{R}^{d}\right)^{N} \cap L^{1}\left(\mathbb{R}^{d}\right)^{N}$. Then the following theorem immediately follows. 
THEOREM 2.1. Let $T>0$. Then there exists a suitable constant $W$ depending on $T$ and on the initial data, such that, if $M$ is a $M M F$ on the interval $I:=\{u \in \mathbb{R}:|u| \leq$ $W\}$, for every $\epsilon>0$ there exists a unique solution $f^{\epsilon} \in C\left([0, T] ; L^{\infty}\left(R^{d}\right)^{N} \cap L^{1}\left(R^{d}\right)^{N}\right)$ to problem (1.2). Moreover the following estimates hold

i)

$$
-C_{i}+M_{i}\left(-\left\|u_{0}\right\|_{L^{\infty}\left(\mathbb{R}^{d}\right)}\right) \leq f_{i}^{\epsilon}(x, t) \leq C_{i}+M_{i}\left(\left\|u_{0}\right\|_{L^{\infty}\left(\mathbb{R}^{d}\right)}\right), \quad 0 \leq t \leq T,
$$

for $i=1, \ldots, N$, where $C_{i}:=W+\beta_{i} \max _{[-W, W]}|G|$;

ii)

$$
\left\|u^{\epsilon}(\cdot, t)\right\|_{L^{\infty}\left(\mathbb{R}^{d}\right)} \leq W
$$

iii) if $f^{\epsilon}$ and $\overline{f^{\epsilon}}$ are two solutions, then, for $0 \leq s \leq t \leq T$,

$$
\sum_{i=1}^{N} \int_{\mathbb{R}^{d}}\left|f_{i}^{\epsilon}(x, t)-\overline{f_{i}^{\epsilon}}(x, t)\right| d x \leq e^{L(t-s)} \sum_{i=1}^{N} \int_{R^{d}}\left|f_{i}^{\epsilon}(x, s)-\overline{f_{i}^{\epsilon}}(x, s)\right| d x,
$$

where $L$ is the Lipschitz constant of $G$ in $[-W, W]$.

3. Convergence. In this section we study the convergence of the sequence $\left\{f^{\epsilon}\right\}$ of solutions of problems (1.2) when $\epsilon$ goes to zero. We prove that a subsequence of $\left\{f^{\epsilon}\right\}$ converges to a limit function $f$, which is a Maxwellian distribution, i.e. $M_{i}(u)=f_{i}$ for $i=1, \ldots, N$, where $u=\sum_{i=1}^{N} f_{i}$ is an entropy solution of problem (1.1).

Let $T>0$, let $W$ be the constant in Theorem 2.1; in the whole section we assume that $M$ is a MMF in the interval $[-W, W]$ and $M_{i}(v)=M_{i}(W \operatorname{sgn} v)$ for $|v|>W, i=1, \ldots, N$.

As a consequence of Proposition 2.2 we have the following one.

Proposition 3.1 (Equicontinuity in $x$ ). Let $T>0, u_{0} \in L^{\infty}\left(\mathbb{R}^{d}\right) \cap L^{1}\left(\mathbb{R}^{d}\right)$, $f_{0 i}^{\epsilon}=M_{i}\left(u_{0}\right)$ and $f^{\epsilon} \in C\left([0, T] ; L^{\infty}\left(\mathbb{R}^{d}\right)^{N} \cap L^{1}\left(\mathbb{R}^{d}\right)^{N}\right)$ be the solution of equation in (1.2) corresponding to $f_{0 i}^{\epsilon}$. Then there exists a positive constant $h_{0}$ and a continuous nondecreasing function $\omega \in C\left(\left(0, h_{0}\right]\right)$, not depending on $\epsilon, \omega(0)=0$, such that, for $0 \leq t \leq T$ and for $h \in \mathbb{R}^{d},|h| \leq h_{0}$

$$
\int_{\mathbb{R}^{d}} \sum_{i=1}^{N}\left|f_{i}^{\epsilon}(x+h, t)-f_{i}^{\epsilon}(x, t)\right| d x \leq \omega(|h|) .
$$

As regard to equicontinuity in $t$ we need the following interpolation lemma due to Kruzkov [10].

LEMMA 3.1. Let $\Omega \subseteq \mathbb{R}^{d}$ be a convex open set and set $\Omega_{h_{0}}=\left\{x \in \mathbb{R}^{d} \mid d(x, \Omega)<\right.$ $\left.h_{0}\right\}$, for some fixed $h_{0}>0$. Let $w$ be a measurable bounded function in $\Omega_{h_{0}} \times$ $(0, T)(T>0)$ and let $\omega_{\Omega} \in C\left(\left[0, h_{0}\right)\right)$ be a nondecreasing function, with $\omega_{\Omega}(0)=0$, such that for $t \in(0, T),|h| \leq h_{0}$

$$
\int_{\Omega_{h_{0}}}|w(x+h, t)-w(x, t)| d x \leq \omega_{\Omega}(|h|) .
$$

Assume the following condition holds:

$$
\left|\int_{\omega}(w(x, t+\tau)-w(x, t)) \varphi(x)\right| \leq C_{\Omega} \tau\|\varphi\|_{C^{2}}
$$


for every $t, t+\tau \in(0, T)(\tau>0)$, for every $\varphi \in C_{0}^{2}(\Omega)$ and some constant $C_{\omega}>0$. Then for $0 \leq t \leq t+\tau \leq T$ we have

$$
\int_{\Omega}|w(x+h, t)-w(x, t)| d x \leq \bar{\omega}_{\Omega}(\tau)
$$

where

$$
\bar{\omega}_{\Omega}(\tau)=C_{\Omega} \min _{|h| \leq h_{0}}\left[|h|+\omega_{\Omega}(|h|)+\frac{\tau}{h^{2}}\right] .
$$

We will use the above lemma to prove the following two propositions.

Proposition 3.2. Let $T>0, u_{0} \in L^{\infty}\left(\mathbb{R}^{d}\right) \cap L^{1}\left(\mathbb{R}^{d}\right), f_{0 i}^{\epsilon}=M_{i}\left(u_{0}\right)$ and $f^{\epsilon} \in C\left([0, T] ; L^{\infty}\left(\mathbb{R}^{d}\right)^{N} \cap L^{1}\left(\mathbb{R}^{d}\right)^{N}\right)$ be the solution of equation in (1.2) corresponding to $f_{0 i}^{\epsilon} ;$ let $u^{\epsilon}:=\sum_{i=1}^{N} f_{i}^{\epsilon}$. Then there exist a positive constant $\tau_{0}$ and a continuous nondecreasing function $\bar{\omega}:\left[0, \tau_{0}\right] \rightarrow \mathbb{R}$, independent on $\epsilon$, with $\bar{\omega}(0)=0$, such that for $0 \leq t \leq t+\tau \leq T, \tau \in\left(0, \tau_{0}\right)$

$$
\int_{\mathbb{R}^{d}}\left|u^{\epsilon}(x, t+\tau)-u^{\epsilon}(x, t)\right| d x \leq \bar{\omega}(\tau) .
$$

Proof. Thanks to Proposition 3.1 and Lemma 3.1 it is sufficient to prove that $u^{\epsilon}$ verifies inequality (3.1). Let $\varphi \in C_{0}^{2}\left(\mathbb{R}^{d}\right)$. Using the property $M_{1}$ of $M$ we have

$$
\begin{gathered}
\left|\int_{\mathbb{R}^{d}}\left(u^{\epsilon}(x, t+\tau)-u^{\epsilon}(x, t)\right) \varphi(x) d x\right| \\
=\left|\sum_{i=1}^{N} \int_{\mathbb{R}^{d}} \int_{t}^{t+\tau}\left(-\sum_{j=1}^{d} \lambda^{i} f_{i}^{\epsilon} \varphi_{x_{j}}+\mu^{i} f_{i}^{\epsilon} \Delta \varphi+\beta_{i} G\left(u^{\epsilon}\right) \varphi\right) d x d s\right| \\
\leq C \tau e^{L(t+\tau)}\|\varphi\|_{C^{2}\left(\mathbb{R}^{d}\right)}\left\|f_{0 i}^{\epsilon}\right\|_{L^{1}\left(\mathbb{R}^{d}\right)},
\end{gathered}
$$

where $C$ is a constant depending on the parameters $\lambda^{i}$ and $\mu^{i}$ and on $L$.

Proposition 3.3. Let $T>0, u_{0} \in L^{\infty}\left(\mathbb{R}^{d}\right) \cap L^{1}\left(\mathbb{R}^{d}\right), f_{0 i}^{\epsilon}=M_{i}\left(u_{0}\right)$ and $f^{\epsilon} \in$ $C\left([0, T] ; L^{\infty}\left(\mathbb{R}^{d}\right)^{N} \cap L^{1}\left(\mathbb{R}^{d}\right)^{N}\right)$ be the solution of equation in (1.2) corresponding to $f_{0 i}^{\epsilon} ;$ let $u^{\epsilon}:=\sum_{i=1}^{N} f_{i}^{\epsilon}$. Then for every $\nu>0$, there exist a positive constant $\tau_{0}$ and $a$ continuous nondecreasing function $\bar{\omega}_{\nu}:\left[0, \tau_{0}\right] \rightarrow \mathbb{R}$, independent on $\epsilon$, with $\bar{\omega}_{\nu}(0)=0$, such that for $\nu \leq t \leq t+\tau \leq T, \tau \in\left(0, \tau_{0}\right)$,

$$
\int_{\mathbb{R}^{d}} \sum_{i=1}^{N}\left|f_{i}^{\epsilon}(x, t+\tau)-f_{i}^{\epsilon}(x, t)\right| d x \leq \bar{\omega}_{\nu}(\tau)
$$

for every $0<\epsilon<\nu$.

Proof. As in the previous proof, we have only to prove inequality (3.1) for $f_{i}^{\epsilon}$, for $\nu \leq t \leq t+\tau \leq T, \tau \in\left(0, \tau_{0}\right)$,. Let $\varphi \in C_{0}^{2}\left(\mathbb{R}^{d}\right)$. If $\mu^{i} \neq 0$ we can use the Green's formula (2.1), as in Proposition 3.1.

We obtain

$$
\begin{aligned}
& \left.\mid \int_{\mathbb{R}^{d}} f_{i}^{\epsilon}(x, t+\tau)-f_{i}^{\epsilon}(x, t)\right) \varphi(x) d x \mid \\
\leq & \mid \int_{\mathbb{R}^{d}} \int_{\mathbb{R}^{d}} f_{0}^{\epsilon}(y)\left(e^{-\frac{t+\tau}{\epsilon}} K_{i}(x-y, t+\tau) \varphi\left(x-\lambda^{i}(t+\tau)\right)\right.
\end{aligned}
$$




$$
\begin{gathered}
\left.-e^{-\frac{t}{\epsilon}} K_{i}(x-y, t) \varphi\left(x-\lambda^{i} t\right)\right) d x d y \mid \\
+\mid \int_{\mathbb{R}^{d}} \int_{0}^{t+\tau} \int_{\mathbb{R}^{d}}\left(e^{-\frac{t+\tau-s}{\epsilon}} K_{i}(x-y, t+\tau-s)\right. \\
\cdot\left(\frac{1}{\epsilon} M_{i}\left(u^{\epsilon}(y, s)\right)+\beta_{i} G\left(u^{\epsilon}(y, s)\right)\right) \varphi\left(x-\lambda^{i}(t+\tau-s)\right. \\
-\int_{\mathbb{R}^{d}} \int_{0}^{t} e^{-\frac{t-s}{\epsilon}} K_{i}(x-y, t-s) \\
\cdot\left(\frac{1}{\epsilon} M_{i}\left(u^{\epsilon}(y, s)\right)+\beta_{i} G\left(u^{\epsilon}(y, s)\right)\right) \varphi\left(x-\lambda^{i}(t-s)\right) d x d s d y \mid \\
=:\left|I_{1}\right|+\left|I_{2}\right| .
\end{gathered}
$$

As regard to $I_{1}$ we have

$$
\left|I_{1}\right| \leq
$$

$\leq\|\varphi\|_{C^{0}}\left\|f_{0}^{\epsilon}\right\|_{L^{1}} \int_{\mathbb{R}^{d}}\left|e^{-\frac{t+\tau}{\epsilon}} K_{i}(x, t+\tau)-e^{-\frac{t}{\epsilon}} K_{i}(x, t)\right| d x$

$$
+e^{-\frac{\nu}{\epsilon}} \tau \sum_{j=1}^{d}\left|\lambda_{j}^{i}\right|\left\|\varphi_{x_{j}}\right\|_{C^{0}}\left\|f_{0 i}^{\epsilon}\right\|_{L^{1}}
$$

Using the expression of $K_{i}$, we obtain for $t \geq \nu$ and $\epsilon \in(0, \nu)$

$$
\int_{\mathbb{R}^{d}}\left|e^{-\frac{t+\tau}{\epsilon}} K_{i}(x, t+\tau)-e^{-\frac{t}{\epsilon}} K_{i}(x, t)\right| \leq \tau\left(\frac{e^{-\frac{\nu}{\epsilon}}}{\nu}+\frac{e^{-\frac{\nu}{\epsilon}}}{\epsilon}\right)=\tau\left(\frac{e^{-\frac{\nu}{\epsilon}}}{\nu}+C_{\nu}\right) .
$$

Hence there exists a positive constant $C$, depending on $\nu$ and $\lambda_{j}^{i}$, such that

$$
\left|I_{1}\right| \leq\|\varphi\|_{C^{1}\left(\mathbb{R}^{d}\right)}\left\|f_{0 i}^{\epsilon}\right\|_{L^{1}\left(\mathbb{R}^{d}\right)} C \tau .
$$

As for $I_{2}$ we have

$$
\begin{aligned}
&\left|I_{2}\right|= \\
&= \mid \int_{-\tau}^{t} \int_{\mathbb{R}^{d}} \int_{\mathbb{R}^{d}} e^{-\frac{t-s}{\epsilon}} K_{i}(x-y, t-s) \varphi\left(x-\lambda^{i}(t-s)\right) \\
& \cdot\left(\frac{1}{\epsilon} M_{i}\left(u^{\epsilon}(x, s+\tau)\right)+\beta_{i} G\left(u^{\epsilon}(x, s+\tau)\right)\right) d x d y d s \\
&-\int_{0}^{t} \int_{\mathbb{R}^{d}} \int_{\mathbb{R}^{d}} e^{-\frac{t-s}{\epsilon}} K_{i}(x-y, t-s) \varphi\left(x-\lambda^{i}(t-s)\right) \\
& \leq \mid \int_{0}^{t} \int_{\mathbb{R}^{d}} \int_{\mathbb{R}^{d}} e^{-\frac{t-s}{\epsilon}} K_{i}(x-y, t-s) \varphi\left(x-\lambda^{i}(t-s)\right) \\
& \cdot\left(\frac{1}{\epsilon} M_{i}\left(u^{\epsilon}(x, s+\tau)\right)+\beta_{i} G\left(u^{\epsilon}(x, s+\tau)\right)-\frac{1}{\epsilon} M_{i}\left(u^{\epsilon}(x, s)\right)+\beta_{i} G\left(u^{\epsilon}(x, s)\right)\right) d x d y d s \mid \\
&+\mid \int_{-\tau}^{0} \int_{\mathbb{R}^{d}} \int_{\mathbb{R}^{d}} e^{-\frac{t-s}{\epsilon}} K_{i}(x-y, t-s) \varphi\left(x-\lambda^{i}(t-s)\right)
\end{aligned}
$$




$$
\cdot\left(\frac{1}{\epsilon} M_{i}\left(u^{\epsilon}(x, s+\tau)\right)+\beta_{i} G\left(u^{\epsilon}(x, s+\tau)\right)\right) d x d y d s|=:| J_{1}|+| J_{2} \mid .
$$

Thanks to the properties of $M$ and $G$, to Proposition 3.1 and to Proposition 2.2 we can obtain the following estimates for $J_{1}$ and $J_{2}$ :

$$
\begin{gathered}
\left|J_{1}\right| \leq \\
\leq \int_{0}^{t}\left(\frac{1}{\epsilon}+L \beta_{i}\right) e^{-\frac{t-s}{\epsilon}} \int_{\mathbb{R}^{d}}\left|\varphi\left(x-\lambda^{i}(t-s)\right)\right|\left|\left(u^{\epsilon}(x, s+\tau)-u^{\epsilon}(x, s)\right)\right| d x d s \\
\leq C_{1} \tau\|\varphi\|_{C^{2}\left(\mathbb{R}^{d}\right)}\left(1-e^{-\frac{t}{\epsilon}}\right) \leq C_{\nu} \tau\|\varphi\|_{C^{2}\left(\mathbb{R}^{d}\right)},
\end{gathered}
$$

where $C_{\nu}$ is a suitable constant depending on $\nu, L$ and $\beta_{i}$;

$$
\begin{gathered}
\left|J_{2}\right| \leq \\
\leq\|\varphi\|_{C^{0}\left(\mathbb{R}^{d}\right)} \int_{-\tau}^{0}\left(\frac{1}{\epsilon}+\beta_{i} L\right) e^{-\frac{t-s}{\epsilon}} \int_{\mathbb{R}^{d}}\left|u^{\epsilon}(x, s+\tau)\right| d x d s \\
\leq\|\varphi\|_{C^{0}\left(\mathbb{R}^{d}\right)} \tau\left(\frac{1}{\epsilon}+\beta_{i} L\right) e^{-\frac{t}{\epsilon}} e^{L \tau} \sum_{i=1}^{N}\left\|f_{0 i}^{\epsilon}\right\|_{L^{1}\left(\mathbb{R}^{d}\right)} \\
\leq C_{\nu, T}\|\varphi\|_{C^{0}\left(\mathbb{R}^{d}\right)} \tau \sum_{i=1}^{N}\left\|f_{0 i}^{\epsilon}\right\|_{L^{1}\left(\mathbb{R}^{d}\right)},
\end{gathered}
$$

where $C_{\nu, T}$ is a suitable constant, depending on $\nu, T, L$, and $\beta_{i}$. Hence

$$
\left|I_{2}\right| \leq\left(C_{\nu}+C_{\nu, T} \sum_{i=1}^{N}\left\|f_{0 i}^{\epsilon}\right\|_{L^{1}\left(\mathbb{R}^{d}\right)}\right)\|\varphi\|_{C^{2}\left(\mathbb{R}^{d}\right)} \tau .
$$

Therefore there exists a positive constant $C=C\left(\nu, T,\left\|f_{0 i}^{\epsilon}\right\|_{L^{1}\left(\mathbb{R}^{d}\right)}, i=1, \ldots, N\right)$ such that

$$
\left|\int_{\mathbb{R}^{d}}\left(f_{i}^{\epsilon}(x, t+\tau)-f_{i}^{\epsilon}(x, t)\right) \varphi(x) d x\right| \leq C\|\varphi\|_{C^{2}\left(\mathbb{R}^{d}\right)} \tau,
$$

for $i=1, \ldots, m$. For $i=m+1, \ldots, N$, the set of hyperbolic equations, the same estimate can be obtained using the Duhamel's formula (see [14]). Summing up the inequalities (3.2) for $i=1, \ldots, N$ we obtain the claim.

Proposition 2.2 and 3.3 allow us to use compacteness method to prove the convergence of a subsequence of $\left\{f^{\epsilon}\right\}$, respectively of $\left\{u^{\epsilon}\right\}$, to some functions $f$ and $u$. The following propositions are necessary to prove that $u$ is an entropy solution to problem (1.2).

Proposition 3.4. Let $T>0, u_{0} \in L^{\infty}\left(\mathbb{R}^{d}\right) \cap L^{1}\left(\mathbb{R}^{d}\right), f_{0 i}^{\epsilon}=M_{i}\left(u_{0}\right)$ and $f^{\epsilon} \in$ $C\left([0, T] ; L^{\infty}\left(\mathbb{R}^{d}\right)^{N} \cap L^{1}\left(\mathbb{R}^{d}\right)^{N}\right)$ be the solution of equation in (1.2) corresponding to $f_{0 i}^{\epsilon} ;$ let $u^{\epsilon}:=\sum_{i=1}^{N} f_{i}^{\epsilon}$. Then, if $u_{0}, u_{0_{x_{k}}} \in B V\left(\mathbb{R}^{d}\right)$ for every $1 \leq k \leq d$, there exists a positive constant $C$ such that, for every $\epsilon<1$ and for every $0 \leq t \leq T$

$$
\sum_{i=1}^{N} \int_{\mathbb{R}^{d}}\left|f_{i}^{\epsilon}-M_{i}\left(u^{\epsilon}\right)\right|
$$


A RELAXATION APPROXIMATION FOR PARABOLIC EQUATIONS

$$
\leq C\left[\sqrt{\epsilon} \sum_{i=1}^{N}\left\|f_{0 i}^{\epsilon}\right\|_{B V\left(\mathbb{R}^{d}\right)}+\epsilon \sum_{i=1}^{N} \sum_{j=1}^{d}\left\|f_{0 i_{x_{j}}}^{\epsilon}\right\|_{B V\left(\mathbb{R}^{d}\right)}\right] .
$$

Proof. Consider the sequences of functions $\left\{u_{0}^{n}\right\}$ regularizing $u_{0}$, and the corresponding sequences of solutions $\left\{u^{\epsilon n}\right\}$ and $\left\{f^{\epsilon n}\right\}$. Let us also set

$$
g_{i}^{\epsilon}(x, t)=f_{i_{x_{k}}}^{\epsilon}, \quad v^{\epsilon}=\sum_{i=1}^{N} g_{i}^{\epsilon}
$$

For $i=1, \ldots, m, g_{i}^{\epsilon}$ satisfies the equation

$$
g_{i_{t}}^{\epsilon}+\sum_{j=1}^{d} \lambda_{j}^{i} g_{i_{x_{j}}}^{\epsilon}-\mu^{i} \Delta g_{i}^{\epsilon}=\frac{1}{\epsilon}\left(M_{i}^{\prime}\left(u^{\epsilon}\right) v^{\epsilon}-g_{i}^{\epsilon}\right)+\beta_{i} G^{\prime}\left(u^{\epsilon}\right) v^{\epsilon}
$$

then, using the Green's formula we obtain

$$
\begin{aligned}
\int_{\mathbb{R}^{d}}\left|g_{i}^{\epsilon}\left(x^{i}+h, t\right)-g_{i}^{\epsilon}\left(x^{i}, t\right)\right| d x^{i} \leq e^{-\frac{t}{\epsilon}} \int_{\mathbb{R}^{d}}\left|g_{0 i}^{\epsilon}(y+h)-g_{0 i}^{\epsilon}(y)\right| d y \\
+\frac{1}{\epsilon} \frac{2 h}{\sqrt{4 \pi \mu^{i}}} \int_{0}^{t} \frac{e^{-\frac{t-s}{\epsilon}}}{\sqrt{t-s}} \int_{\mathbb{R}^{d}} M_{i}^{\prime}\left(u^{\epsilon}\left(y+\lambda^{i} s, s\right)\left|v^{\epsilon}\left(y+\lambda^{i} s, s\right)\right| d y d s\right. \\
+\beta_{i} \frac{2 h}{\sqrt{4 \pi \mu^{i}}} \int_{0}^{t} \frac{e^{-\frac{t-s}{\epsilon}}}{\sqrt{t-s}} \int_{\mathbb{R}^{d}} \mid G^{\prime}\left(u^{\epsilon}\left(y+\lambda^{i} s, s\right)|| v^{\epsilon}\left(y+\lambda^{i} s, s\right) \mid d y d s .\right.
\end{aligned}
$$

Summing up the above inequalities for $i=1, \ldots, m$ we obtain

$$
\begin{gathered}
\sum_{i=1}^{m} \int_{\mathbb{R}^{d}}\left|g_{i}^{\epsilon}\left(x^{i}+h, t\right)-g_{i}^{\epsilon}\left(x^{i}, t\right)\right| d x \leq e^{-\frac{t}{\epsilon}} \sum_{i=1}^{m} \int_{\mathbb{R}^{d}}\left|g_{0 i}^{\epsilon}\left(x^{i}+h, t\right)-g_{0 i}^{\epsilon}\left(x^{i}, t\right)\right| d x \\
+\left(\frac{1}{\epsilon}+L\right) \frac{2 h}{\sqrt{4 \pi \mu}} \int_{0}^{t} \frac{e^{-\frac{t-s}{\epsilon}}}{\sqrt{t-s}} \sum_{i=1}^{N} \int_{\mathbb{R}^{d}}\left|f_{i_{x_{k}}}^{\epsilon}\right| d x,
\end{gathered}
$$

where $\mu:=\min \left\{\mu_{1}, \ldots, \mu_{m}\right\}$.

Thanks to the uniform (in $\epsilon$ ) estimate for the BV - norm of $f_{i}^{\epsilon n}$, coming from inequality (2.3), we obtain the following inequality

$$
\sum_{i=1}^{m} \int_{\mathbb{R}^{d}}\left|f_{i_{x_{k}}}^{\epsilon n}(x+h, t)-f_{i_{x_{k}}}^{\epsilon n}(x, t)\right| d x \leq e^{-\frac{t}{\epsilon}} \sum_{i=1}^{m} \int_{\mathbb{R}^{d}}\left|f_{0 i_{x_{k}}}^{n}(x+h, t)-f_{0 i_{x_{k}}}^{n}(x)\right| d x
$$

$$
+\frac{2 h}{\sqrt{2 \mu}} e^{L T} \sum_{i=1}^{N}\left\|f_{0 i}^{n}\right\|_{B V\left(\mathbb{R}^{d}\right)}\left(\frac{1}{\sqrt{\epsilon}}+L \sqrt{\epsilon}\right) .
$$

Now we use the equation for $f_{i}^{\epsilon n}$ to obtain

$$
\partial_{t}\left|f_{i}^{\epsilon n}-M_{i}\left(u^{\epsilon n}\right)\right|+\frac{1}{\epsilon}\left|f_{i}^{\epsilon n}-M_{i}\left(u^{\epsilon n}\right)\right| \leq C_{0}\left(\sum_{i=1}^{N} \sum_{j=1}^{d}\left|f_{i_{x_{j}}}^{\epsilon n}\right|+\sum_{i=1}^{m} \sum_{j=1}^{d}\left|f_{i_{x_{j} x_{j}}}^{\epsilon n}\right|\right),
$$


for a suitable constant $C_{0}$; hence we have

$$
\int_{\mathbb{R}^{d}} \sum_{i=1}^{N}\left|f_{i}^{\epsilon n}-M_{i}\left(u^{\epsilon n}\right)\right| d x \leq C_{1} \int_{0}^{t} \int_{\mathbb{R}^{d}} e^{-\frac{t-s}{\epsilon}}\left(\sum_{i=1}^{N} \sum_{j=1}^{d}\left|f_{i_{x_{j}}}^{\epsilon n}\right|+\sum_{i=1}^{m} \sum_{j=1}^{d}\left|f_{i_{x_{j} x_{j}}}^{\epsilon n}\right|\right)
$$

and using the estimates for the $B V$-norms of $f_{i}^{\epsilon n}$ and $f_{i_{x_{j}}}^{\epsilon n}$, coming from (2.3) and (3.3) we obtain

$$
\int_{\mathbb{R}^{d}} \sum_{i=1}^{N}\left|f_{i}^{\epsilon n}-M_{i}\left(u^{\epsilon n}\right)\right| d x \leq C_{2}\left[\sqrt{\epsilon} \sum_{i=1}^{N}\left\|f_{0 i}^{\epsilon n}\right\|_{B V\left(\mathbb{R}^{d}\right)}+\epsilon \sum_{i=1}^{m} \sum_{j=1}^{d}\left\|f_{0 i_{x_{j}}}^{\epsilon n}\right\|_{B V\left(\mathbb{R}^{d}\right)}\right],
$$

where $C_{1}$ and $C_{2}$ are suitable constants. By standard density arguments we obtain the claim.

Proposition 3.5. Let $T>0, u_{0} \in L^{\infty}\left(\mathbb{R}^{d}\right) \cap L^{1}\left(\mathbb{R}^{d}\right), f_{0 i}^{\epsilon}=M_{i}\left(u_{0}\right)$ and $f^{\epsilon} \in$ $C\left([0, T] ; L^{\infty}\left(\mathbb{R}^{d}\right)^{N} \cap L^{1}\left(\mathbb{R}^{d}\right)^{N}\right)$ be the solution of equation in (1.2) corresponding to $f_{0 i}^{\epsilon} ;$ let $u^{\epsilon}:=\sum_{i=1}^{N} f_{i}^{\epsilon}$. Then, for all $k \in \mathbb{R}$ and for all $\varphi \in C_{0}^{\infty}(\mathbb{R} \times[0, T]), \varphi>0$, for $1 \leq i \leq N$ we have

$$
\begin{gathered}
\int_{0}^{T} \int_{\mathbb{R}^{d}}\left(\sum_{i=1}^{N}\left|f_{i}^{\epsilon}-M_{i}(k)\right|\left(\varphi_{t}+\lambda^{i} \varphi_{x}+\mu^{i} \varphi_{x x}\right)+G\left(u^{\epsilon}\right) \varphi\right) d x d t \\
\geq \frac{1}{\epsilon} \int_{0}^{T} \int_{\mathbb{R}^{d}}\left(\sum_{i=1}^{N}\left|f_{i}^{\epsilon}-M_{i}(k)\right|-\left|u^{\epsilon}-k\right|\right) \varphi d x d t \geq 0 .
\end{gathered}
$$

Proof. Multiplying for $\operatorname{sgn}\left(f_{i}^{\epsilon}-M_{i}(k)\right) \varphi$ the equation for $f_{i}^{\epsilon}$ and integrating and summing for $i=1, \ldots, N$ we obtain

$$
\begin{gathered}
\int_{0}^{T} \int_{\mathbb{R}^{d}} \sum_{i=1}^{N}\left|f_{i}^{\epsilon}-M_{i}(k)\right|\left(\varphi_{t}+\lambda^{i} \varphi_{x}+\mu^{i} \varphi_{x x}\right) d x d t \\
=\frac{1}{\epsilon} \int_{0}^{T} \int_{\mathbb{R}^{d}} \sum_{i=1}^{N}\left[\left|f_{i}^{\epsilon}-M_{i}(k)\right|-\operatorname{sgn}\left(f_{i}^{\epsilon}-M_{i}(k)\right)\left(M_{i}\left(u^{\epsilon}\right)-M_{i}(k)\right)\right] \varphi d x d t \\
-\int_{0}^{T} \int_{\mathbb{R}^{d}} G\left(u^{\epsilon}\right) \varphi d x d t .
\end{gathered}
$$

Since

$$
\sum_{i=1}^{N} \operatorname{sgn}\left(f_{i}^{\epsilon}-M_{i}(k)\left(M_{i}\left(u^{\epsilon}\right)-M_{i}(k)\right) \leq\left|u^{\epsilon}-k\right| \leq \sum_{i=1}^{N}\left|f_{i}^{\epsilon}-M_{i}(k)\right|\right.
$$

we obtain the claim.

Now we can prove the following convergence theorem.

Theorem 3.1 (Convergence). Let $T>0, u_{0} \in L^{\infty}\left(\mathbb{R}^{d}\right) \cap L^{1}\left(\mathbb{R}^{d}\right), f_{0 i}^{\epsilon}=$ $M_{i}\left(u_{0}\right)$ and $f^{\epsilon} \in C\left([0, T] ; L^{\infty}\left(\mathbb{R}^{d}\right)^{N} \cap L^{1}\left(\mathbb{R}^{d}\right)^{N}\right)$ be the solution of equation in (1.2) corresponding to $f_{0 i}^{\epsilon}$; let $u^{\epsilon}:=\sum_{i=1}^{N} f_{i}^{\epsilon}$. Then there exists a siubsequence $\left\{f^{\epsilon_{k}}\right\}$ of $\left\{f^{\epsilon}\right\}$ such that

$$
f_{i}^{\epsilon_{k}} \rightarrow f \quad \text { in } \quad C\left([0, T] ; L_{l o c}^{1}\left([0, T] \times R^{d}\right)^{N}\right), \quad \text { for } i=1, \ldots, N,
$$




$$
u^{\epsilon_{k}} \rightarrow u \quad \text { in } \quad C\left([0, T] ; L_{l o c}^{1}\left(R^{d}\right)\right),
$$

where $u=\sum f_{i}^{\epsilon}$ is an entropy solution of problem (1.2).

Proof. From Propositions 2.2 and 3.3 we obtain the relatively compactness of $\left\{f^{\epsilon}\right\}$ in $C\left([\nu, T] ; L^{1}\left(\mathbb{R}^{d}\right)^{N}\right)$, for every $\nu>0$; then there exists a subsequence, also denoted by $\left\{f^{\epsilon}\right\}$, converging in $C\left([\nu, T] ; L_{l o c}^{1}\left(\mathbb{R}^{d}\right)^{N}\right)$ to some $f$.

In order to prove that the convergence holds also in $t=0$, we consider a sequence $\left\{u_{0 n}\right\} \subset C_{0}^{\infty}\left(\mathbb{R}^{d}\right)$ converging to $u_{0}$ in $L^{1}\left(\mathbb{R}^{d}\right)$ and the corresponding solutions $u^{\epsilon n}$ and $f^{\epsilon n}$. We easily obtain the following estimates

$$
\begin{gathered}
\sum_{i=1}^{N}\left\|f_{0 i}^{\epsilon n}\right\|_{B V\left(\mathbb{R}^{d}\right)} \leq c_{1} n \sum_{i=1}^{N}\left\|f_{0 i}\right\|_{L^{1}\left(\mathbb{R}^{d}\right)}, \\
\sum_{i=1}^{m}\left\|f_{0 i_{x_{j}}}^{\epsilon n}\right\|_{B V\left(\mathbb{R}^{d}\right)} \leq c_{2} n^{2} \sum_{i=1}^{m}\left\|f_{0 i}\right\|_{L^{1}\left(\mathbb{R}^{d}\right)}, \quad j=1, \ldots, d,
\end{gathered}
$$

where $c_{1}$ and $c_{2}$ are suitable constants. For every $\epsilon<1$, we choose $n=\epsilon^{-\frac{1}{4}}$ in the following inequality

$$
\begin{gathered}
\sum_{i=1}^{N} \int_{\mathbb{R}^{d}}\left|M_{i}\left(u^{\epsilon}\right)-f_{i}^{\epsilon}\right| \\
\leq \sum_{i=1}^{N} \int_{\mathbb{R}^{d}}\left|f_{i}^{\epsilon}-f_{i}^{\epsilon n}\right|+\sum_{i=1}^{N} \int_{\mathbb{R}^{d}}\left|f_{i}^{\epsilon n}-M_{i}\left(u^{\epsilon n}\right)\right|+\sum_{i=1}^{N} \int_{\mathbb{R}^{d}}\left|M_{i}\left(u^{\epsilon n}\right)-M_{i}\left(u^{\epsilon}\right)\right|,
\end{gathered}
$$

and using Proposition 3.4, (3.5) and (3.6) we obtain that

$$
\lim _{\epsilon \rightarrow 0} \sum_{i=1}^{N} \int_{\mathbb{R}^{d}}\left|M_{i}\left(u^{\epsilon}\right)-f_{i}^{\epsilon}\right|=0 .
$$

Now the convergence of $f^{\epsilon}$ to $f$ in $C\left([0, T] ; L^{1}\left(\mathbb{R}^{d}\right)\right)$ easily follows from the inequality

$$
\sup _{[0, T]} \int_{\mathbb{R}^{d}}\left|f_{i}^{\epsilon}-f_{i}\right| \leq \sup _{[0, T]} \int_{\mathbb{R}^{d}}\left|f_{i}^{\epsilon}-M_{i}\left(u^{\epsilon}\right)\right|+\sup _{[0, T]} \int_{\mathbb{R}^{d}}\left|M_{i}\left(u^{\epsilon}\right)-M_{i}(u)\right|,
$$

where $u:=\sum_{i=1}^{N} f_{i}$.

Now we have to prove that the function $u$ verifies the conditions (E). To this purpose we remark that

$$
\begin{gathered}
\sum_{i=1}^{N}\left|f_{i}-M_{i}(k)\right|=\sum_{i=1}^{N}\left|M_{i}(u)-M_{i}(k)\right|=|u-k| \\
\quad \text { for a.e. }(x, t) \in \mathbb{R}^{d} \times(0,+\infty), \\
\sum_{i=1}^{N} \lambda_{j}^{i}\left|f_{i}-M_{i}(k)\right|=\sum_{i=1}^{N} \lambda_{j}^{i} \operatorname{sgn}(u-k)\left(M_{i}(u)-M_{i}(k)\right)=\operatorname{sgn}(u-k)\left(A_{j}(u)-A_{j}(k)\right) \\
\quad \text { for a.e. }(x, t) \in \mathbb{R}^{d} \times(0,+\infty), \text { for } j=1, \ldots, d, \\
\sum_{i=1}^{N} \mu_{i}\left|f_{i}-M_{i}(k)\right|=\sum_{i=1}^{N} \mu_{i} \operatorname{sgn}(u-k)\left(M_{i}(u)-M_{i}(k)\right)=\operatorname{sgn}(u-k)(B(u)-B(k))
\end{gathered}
$$


for a.e. $(x, t) \in \mathbb{R}^{d} \times(0,+\infty)$.

Now the conclusion follows letting $\epsilon$ go to zero in (3.4).

4. Examples.

EXAMPLE 1. Consider the equation

$$
u_{t}+A(u)_{x}-u_{x x}=0 \quad \text { in } \mathbb{R} \times \mathbb{R}^{+} ;
$$

since the function $B$ is the identity, it is sufficient to set $N=2$ in order to build a relaxation approximation system. We set

$$
\begin{gathered}
\lambda^{1}=-\alpha, \quad \lambda^{2}=\alpha, \quad \alpha>0, \\
\mu^{i}=1 \quad \text { for } i=1,2 .
\end{gathered}
$$

Now conditions $\left(M_{1}\right)-\left(M_{3}\right)$ give

$$
M_{1}(u)=\frac{1}{2}\left[u-\frac{A(u)}{\alpha}\right], \quad M_{2}(u)=\frac{1}{2}\left[u+\frac{A(u)}{\alpha}\right]
$$

and the quasimonotonicity conditions implies the following limitation to $\alpha$

$$
\sup _{I}\left|A^{\prime}(u)\right| \leq \alpha
$$

Set $v^{\epsilon}:=\alpha\left(f_{2}^{\epsilon}-f_{1}^{\epsilon}\right)$; then we obtain the relaxation system

$$
\left\{\begin{array}{l}
u_{t}^{\epsilon}+v_{x}^{\epsilon}-u_{x x}^{\epsilon}=0 \\
v_{t}^{\epsilon}+\alpha^{2} u_{x}^{\epsilon}-v_{x x}^{\epsilon}=\frac{1}{\epsilon}\left(A(u)^{\epsilon}-v^{\epsilon}\right)
\end{array}\right.
$$

which is an extension to parabolic case of the scheme in [8] and of the Example 4.1 in [14].

EXAMPLE 2. Consider now the quasilinear parabolic equation

$$
\begin{aligned}
u_{t}+A(u)_{x}-(B(u))_{x x} & =0 \quad \text { in } \mathbb{R} \times \mathbb{R}^{+}, \\
B^{\prime}(u) & \geq 0 .
\end{aligned}
$$

Set $N=3$ and

$$
\lambda^{1}=-\alpha, \quad \lambda^{2}=0, \quad \lambda^{3}=\alpha, \quad \mu^{1}=0, \quad \mu^{2}=\alpha, \quad \mu^{3}=0, \quad \alpha>0 .
$$

We obtain

$$
\begin{aligned}
M_{1}(u)= & \frac{1}{2}\left[u-\frac{A(u)}{\alpha}-\frac{B(u)}{\alpha}\right] \\
& M_{2}(u)=\frac{B(u)}{\alpha} \\
M_{3}(u)= & \frac{1}{2}\left[u+\frac{A(u)}{\alpha}-\frac{B(u)}{\alpha}\right]
\end{aligned}
$$

and the quasimonotonicity is ensured by the condition

$$
\alpha \geq \max _{I}\left(B^{\prime}+\left|A^{\prime}\right|\right) \text {. }
$$

Setting

$$
v^{\epsilon}:=\sum_{i=1}^{N} \lambda^{i} f_{i}^{\epsilon}, \quad w^{\epsilon}:=\sum_{i=1}^{N} \mu^{i} f_{i}^{\epsilon}
$$


we obtain

$$
\left\{\begin{array}{l}
u_{t}^{\epsilon}+v_{x}^{\epsilon}-w_{x x}^{\epsilon}=0 \\
v_{t}^{\epsilon}+\left(\alpha^{2} u^{\epsilon}-\alpha w^{\epsilon}\right)_{x}=\frac{1}{\epsilon}\left[A\left(u^{\epsilon}\right)-v^{\epsilon}\right] \\
w_{t}^{\epsilon}-\alpha^{2} w_{x x}^{\epsilon}=\frac{1}{\epsilon}\left[B\left(u^{\epsilon}\right)-w^{\epsilon}\right]
\end{array}\right.
$$

In the case $A(u)=0$ we can set

$$
N=2, \quad \mu^{1}=\gamma, \quad \mu^{2}=\alpha, \quad \gamma \geq \alpha \geq 0
$$

and we obtain

$$
\begin{gathered}
M_{1}(u)=\frac{\alpha u-B(u)}{\alpha-\gamma}, \\
M_{2}(u)=\frac{B(u)-\gamma u}{\alpha-\gamma}, \\
\gamma \leq \min _{I} B^{\prime}(u) \leq \max _{I} B^{\prime}(u) \leq \alpha .
\end{gathered}
$$

If the parabolic term does not degenerate, i.e. $B^{\prime}(u) \geq m>0$, then $\gamma$ and $\alpha$ can be strictly positive; setting

$$
v^{\epsilon}=\sum_{i=1}^{N} \mu^{i} f_{i}^{\epsilon}
$$

we obtain the relaxation system

$$
\left\{\begin{array}{l}
u_{t}^{\epsilon}-v_{x x}^{\epsilon}=0 \\
v^{\epsilon}-\left[(\gamma+\alpha) v^{\epsilon}-\alpha \gamma u^{\epsilon}\right]_{x x}=\frac{1}{\epsilon}\left[\left(B\left(u^{\epsilon}\right)-v^{\epsilon}\right] .\right.
\end{array}\right.
$$

If the condition on the derivative of $B$ is $B^{\prime}(u) \geq 0$, then $\alpha$ has to be zero, and the relaxation system has the form

$$
\left\{\begin{array}{l}
u_{t}^{\epsilon}+v_{x x}^{\epsilon}=0 \\
v_{t}^{\epsilon}-\gamma v_{x x}^{\epsilon}=\frac{1}{\epsilon}\left(B\left(u^{\epsilon}\right)-v^{\epsilon}\right) .
\end{array}\right.
$$

EXAMPLE 3. Now we consider the equation with source term

$$
u_{t}+A(u)_{x}-(B(u))_{x x}=G(u) \quad \text { in } \mathbb{R} \times \mathbb{R}^{+},
$$

where $B^{\prime}(u) \geq 0$.

We can approximate the equation with the relaxation system

$$
\left\{\begin{array}{l}
f_{t}^{1}-\alpha f_{x}^{1}=\frac{1}{\epsilon}\left[\frac{1}{2}\left(u-\frac{A(u)+B(u)}{\alpha}\right)-f^{1}\right]+\frac{G(u)}{2} \\
f_{t}^{2}-\alpha f_{x x}^{2}=\frac{1}{\epsilon}\left[\frac{B(u)}{\alpha}-f^{2}\right] \\
f_{t}^{3}+\alpha f_{x}^{3}=\frac{1}{\epsilon}\left[\frac{1}{2}\left(u+\frac{A(u)-B(u)}{\alpha}\right)-f^{3}\right]+\frac{G(u)}{2}
\end{array}\right.
$$

obtained setting

$$
\lambda^{1}=-\alpha, \quad \lambda^{2}=0, \quad \lambda^{3}=\alpha, \quad \mu^{1}=0, \quad \mu^{2}=\alpha, \quad \mu^{3}=0, \quad \alpha>0,
$$

as for the equation (4.1) and

$$
\beta_{1}=\beta_{3}=\frac{1}{2}, \quad \beta_{2}=0 .
$$


In this case the monotonicity conditions

$$
M_{i}^{\prime}(u) \geq \max \left\{0,-\epsilon \beta_{i} G^{\prime}(u)\right\}
$$

are satisfyed for $\epsilon \leq \epsilon_{0}$ if we set

$$
\alpha \geq \frac{\max _{I}\left(B^{\prime}(u)+\left|A^{\prime}(u)\right|\right)}{1-\epsilon_{0}\left|\min _{I} G^{\prime}(u)\right|},
$$

where $\epsilon_{0}$ is such that $\epsilon_{0}\left|\min _{I} G^{\prime}(u)\right|<1$.

Setting

$$
\begin{aligned}
& v^{\epsilon}:=\sum_{i=1}^{N} \lambda^{i} f_{i}^{\epsilon}, \\
& w^{\epsilon}:=\sum_{i=1}^{N} \mu^{i} f_{i}^{\epsilon},
\end{aligned}
$$

we obtain the conservation form

$$
\left\{\begin{array}{l}
u_{t}^{\epsilon}+v_{x}^{\epsilon}-w_{x x}^{\epsilon}=G\left(u^{\epsilon}\right) \\
v_{t}^{\epsilon}+\left(\alpha^{2} u^{\epsilon}-\lambda w^{\epsilon}\right)_{x}=\frac{1}{\epsilon}\left(A\left(u^{\epsilon}\right)-v^{\epsilon}\right) \\
w_{t}^{\epsilon}-\alpha^{2} w_{x x}^{\epsilon}=\frac{1}{\epsilon}\left(B\left(u^{\epsilon}\right)-w^{\epsilon}\right)
\end{array}\right.
$$

EXAMPLE 4. Let us consider the multidimensional equation

$$
\begin{gathered}
u_{t}-\sum_{j=1}^{d} \partial_{x_{j}} A_{j}(u)-\Delta B(u)=0 \quad \text { in } \mathbb{R}^{d} \times \mathbb{R}^{+}, \\
B^{\prime}(u) \geq 0 ;
\end{gathered}
$$

we set $N=d+2$ and

$$
\begin{gathered}
\lambda_{i}^{i}=-\alpha, \text { for } i=1, \ldots, d, \quad \alpha>0, \\
\lambda_{j}^{N}=\alpha, \text { for } j=1, \ldots, d, \\
\lambda_{j}^{i}=0 \text { otherwise } ; \\
\mu^{i}=\gamma \text { for } i \neq d+1, \quad \gamma \geq 0, \\
\mu^{d+1}=\theta, \quad \theta>0 .
\end{gathered}
$$

The compatibility conditions imply that

$$
\begin{aligned}
& M_{j}(u)=\frac{1}{(d+1)}\left(\frac{(\theta u-B(u))}{\theta-\gamma}+\sum_{i=1}^{d} \frac{A_{i}(u)}{\alpha}\right)-\frac{A_{j}(u)}{\alpha}, \\
& \quad j=1, \ldots, d, \\
& M_{d+1}(u)=\frac{B(u)-\gamma u}{\theta-\gamma}, \\
& M_{N}(u)=\frac{1}{(d+1)(\theta-\gamma)}\left(\theta u-B(u)+(\theta-\gamma) \sum_{j=1}^{d} \frac{A_{j}(u)}{\alpha}\right)
\end{aligned}
$$


and the monotonicity conditions are

$$
0 \leq \gamma<\min _{I} B^{\prime}(u), \quad \theta>2 \max _{I} B^{\prime}(u), \quad \alpha>2(d+1) \max _{I} \sum_{j=1}^{d}\left|A_{j}^{\prime}(u)\right| .
$$

If $B^{\prime}(u) \geq m>0$ then we can choose $\gamma>0$ and we obtain the following relaxation system, where all the equations are of parabolic type

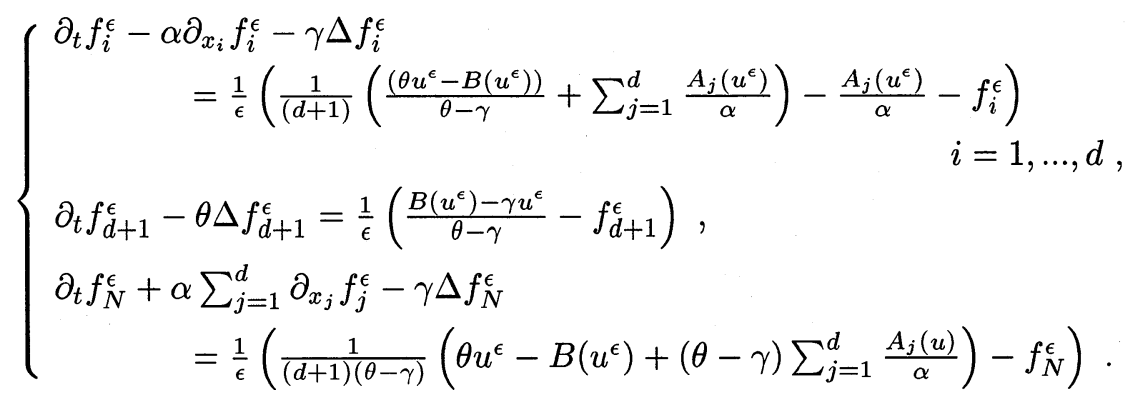

If the parabolic term degenerates, $\gamma$ has to be zero to verify the monotonicity conditions; therefore, as a consequence of the degeneration, the system contains both parabolic and hyperbolic equations.

\section{REFERENCES}

[1] M. Bertsch, P. De Mottoni, And L. Peletier, Degenerate diffusion and the Stefan problem, Nonlinear Analysis TMA, 8 (1984), pp. 1311-1336.

[2] P. BÉNilan AND H. Touré, Sur l'équation générale $u_{t}=a\left(\cdot, u, \varphi(\cdot, u)_{x}\right)_{x}+v$ dans $L^{1}:$ II Le problème d'évolution, Ann. Inst. Henri Poincaré, 12:6 (1995), pp. 727-761.

[3] Y. BREnier, Résolution d'équations d'évolutions quasilinéaires en dimension $N$ d'espace à l'aide d'équations linéaires en dimension $N+1$, J. Diff. Eq., 50 (1983), pp. 375-390.

[4] C. Cercignani, The Boltzmann Equation and Its Applications, Springer Verlag, New York, 1988.

[5] C. Cercignani, R. Illner, and M. Pulvirenti, The Mathematical Theory of Diluite Gases, Springer Verlag, New York, 1994.

[6] E. Di Benedetto, Degenerate Parabolic Equations, Springer Verlag, New York, 1993.

[7] Y. Giga AND T. MiYAKAWA, A kinetic construction of global solution of first order quasilinear equation, Duke Math.J., 50 (1983), pp. 505-515.

[8] S. JiN AND Z. XIN, The relaxation schemes for systems of conservation laws in arbitrary space dimension, Comm. Pure Appl. Math, 48 (1995), pp. 235-277.

[9] B. HANOUZET AND R. NATAlini, Weakly coupled systems of quasilinear hyperbolic equations, Diff. Integral Eq., 9:6 (1996), pp. 1279-1392.

[10] S. N. KRUZǨov, First order quasilinear equations in several independent variables, Mat. Sb., 81 (1970), pp. 228-255; Math. USSR Sb, 10 (1970), pp. 217-243.

[11] P. L. Lions, B. Perthame, AND E. TADMor, A kinetic formulation of multidimensional scalar conservation laws and related equations, Journal A.M.S., 7 (1994), pp. 169-191.

[12] P. MARCATI, Weak solutions to a nonlinear partial differential equation of mixed type, Diff. Integral Eq., 9 (1996), pp. 827-848.

[13] R. NATALINI, Convergence to equilibrium for the relaxation approximations of conservation laws, Comm. Pure Appl. Math., 49:8 (1996), pp. 795-823.

[14] R. NAtAlini, A discrete kinetic approximation of entropy solutions to multidimensional scalar conservation laws, J. Differential Equations, 148 (1998), pp. 292-317.

[15] B. Perthame AND E. TADMOR, A kinetic equation with kinetic entropy functions for scalar conservation laws, Comm. Math. Phys., 136 (1991), pp. 501-517.

[16] M. Protter and H. Weinberger, Maximum Principles in Differential Equations, PrenticeHall, Englewood Cliffs, N.J., 1967. 
[17] A. I. Volpert and S. I. Hudjaev, Cauchy Problem for Degenerate Second Order Quasilinear Parabolic Equations, Math. USSR Sbornik 7, pp. 365-387, Math. Sbornik t., 89 (1969), (in Russian).

[18] J. CARRILLO, Entropy solutions for nonlinear degenerate problems, To appear. 